\title{
Computer Vision based Currency Classification System
}

\author{
Bhupendra Singh \\ Indian Institute of \\ Information Technology \\ Allahabad, India
}

\author{
Pankaj Badoni \\ Indian Institute of \\ Information Technology \\ Allahabad, India
}

\author{
Kuldeep Verma \\ Indian Institute of \\ Information Technology \\ Allahabad, India
}

\begin{abstract}
There are numerous problems associated with current system which are solving the problem of automatic currency classification. Some of the problems administered are like scaling, rotation and noise in the form of missing valuable data in printing or due to the wear and tear of currency notes. In our system we are first aligning the image horizontally along the $\mathrm{x}$ axis and after that foreground of the image is removed by detecting the location of edges, and once we have got the processed image we can apply any of the techniques for classification. Over here we are using fast template matching for recognizing the value of the currency. Once we get result after template matching we can classify the currency into different categories like 10,50,100,500 and 1000. In our system we are aiming at the improvement on existing system by adding useful and robust pre-processing techniques which has been missing in most of the recent works done so far.
\end{abstract}

\section{Keywords:}

Rotation invariant, background removal, correlation, template matching.

\section{INTRODUCTION:}

India is very large country and the way here currency notes are printed it is very difficult for the human operators to manually count money and categorize it into different categories like $1,2,5,10,50,100,500$ and 1000. The main financial institution of India i.e. RBI (Reserve Bank of India) has given some guidelines for identification of Indian currency notes [10], [11].Since the currency is made up of paper, so it is quite prone to wear and tear, and the major hurdle in classification of currency notes is that of the noise which comes in form of torn notes and other factors. Table 1. Suggests the number of currency notes issued in the number of successive years.

Table 1:

\begin{tabular}{|c|c|c|c|c|c|c|}
\hline \multirow[t]{2}{*}{ Denomination } & \multicolumn{3}{|c|}{ Volume(Million Pieces) } & \multicolumn{3}{|c|}{ Value (Rupee Crore) } \\
\hline & $\begin{array}{l}\text { End-March } \\
2006\end{array}$ & $\begin{array}{l}\text { End-March } \\
2007\end{array}$ & $\begin{array}{l}\text { End-March } \\
2008\end{array}$ & $\begin{array}{l}\text { End-March } \\
2006\end{array}$ & $\begin{array}{l}\text { End-March } \\
2007\end{array}$ & $\begin{array}{l}\text { End-March } \\
2008\end{array}$ \\
\hline 1 & 2 & 3 & 4 & 5 & 6 & 7 \\
\hline Rs. 2 and 5 & $6,21 ?$ & 6,008 & 7,405 & 2,431 & 2,334 & 2,747 \\
\hline Rs. 10 & 6,274 & 7,155 & 9,333 & 6,274 & 7,155 & 9,333 \\
\hline Rs. 20 & 2,036 & 2,089 & 2,054 & 4,076 & 4,178 & 4,108 \\
\hline Rs.50 & 5,568 & 5,590 & 5,302 & 27,840 & 27,951 & 26,508 \\
\hline Rs.100 & 13,464 & 13,544 & 13,457 & $1,34,640$ & $1,35,444$ & $1,34,575$ \\
\hline Rs. 500 & 3,647 & 4,508 & 5,262 & $1,82,350$ & $2,25,400$ & $2,63,108$ \\
\hline Rs. 1000 & 643 & 937 & 1,412 & 64,300 & 93676 & $1,41,219$ \\
\hline Total & 37849 & 39,831 & 44,225 & $4,21,911$ & $4,96,138$ & $5,81,598$ \\
\hline
\end{tabular}

In many of approaches which various people have applied in the recent years ,neural network has been quite popular among the classification technique, in one of the system two images are given as input and based on that images it gives output, it's accuracy is up to $90 \%$ [4]. The other person have applied MLP (multi layer perceptron) and it has given some useful results [1], [3]. Only problem with MLP is that it gives best results for only known classes, for any new pattern it gives false result. In another of the methods the where dimensionality of the feature vectors have to be reduced and pattern recognition has to be achieved, for that PCA (principal component analysis) is being used [6], [7].

Since the currency classification is two step process where first the currency is recognized and then it is verified. In above text the methods discussed were mainly focused on the image recognition. Takeda et al. [2] have devised approach for verification, for that statistical method is being employed. This classification was giving good responses but it was not able to solve when the data is heavily co-related. F'rosini et al. [8] have applied the MLP technique for the currency recognition and classification. For recognition MLP uses simple information extracted from image, and for verification it uses pyramidal MLP as autoassociator [9]. In all the techniques which have been discussed so far they give good results but still they work under some constraints, we need to make our system robust and dynamic which is able to classify the currency notes accurately.

Template matching is one of the most useful techniques in image and signal processing, among various applications image recognition, retrieval and detection are the best ones. For a given input and template image, the algorithm finds the partial image that matches the template image in terms of specific criterion, like correlation or Euclidean distance. Over the years many people have given robust and efficient techniques for template matching which reduces complexity and computational cost of matching,[12],[13], in this they have used a low resolution image template for coarse matching, once the template matches in coarse matching then they go ahead with high resolution matching.

[14] B. P. Flannery, et al, used the Fourier transform to perform the correlation operation. With cross correlation of the template image and every part of the input image can be obtained the same time, the computational cost required by all these methods is usually very high by conventional methods of obtaining the cross correlation. Instead if we approximate the image by a corresponding function the computation can be decreased. Schweitzer et al. proposed an efficient template matching algorithm by introducing integral images and approximating the input image with second- or thirdorder polynomials [15].While Shinichiro and Masako Omachi [16] had proposed a fast template matching scheme using Legendre polynomial for approximating the template and then calculating the normalized cross correlation between the input image and sample templates of different sizes. We had considered the work of Shinichiro el al in particular for the task of template matching. 


\section{METHODOLOGY:}

Before we proceed with our methodology and our approach we need to understand why we needed this improvement over existing systems. Since this system is focusing on the currency classification, so the first main requirement is of image acquisition for currency classification, for that we are using the scanned images and also they can be obtained by taking high resolution photographs of currency notes. The issues which are creating problems for the current system are like rotational invariant images, noise in the form of extra background. So these problems have to be taken care of, if we want our system to be robust and highly efficient.

In the current system we are doing the following steps

1. Pre processing

1.1 Rotation invariance

1.2 Background removal.

1.3 Image rescaling.

2. Cross Correlation of given note with sample templates.

3. Classification.

\subsection{Pre-processing for rotation invariance:}

Step 1: Firstly, the canny edge detection Algorithm. Is employed for finding the edges of the note, which will give binary image (fig2). Next, starting from the upper left corner loops are iterated such that for every row the first non-zero pixel location along the columns is find out. This process is repeated until the end of the note/ bottom right corner is reached. The resulting image will be having two lines of the note outer boundary ( it may also contain some pixels outside the note boundary) as shown in fig 3

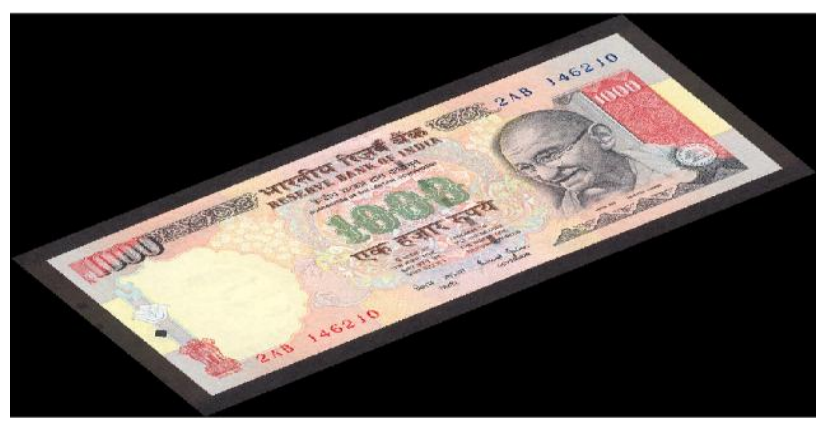

Figure 1: image input to system

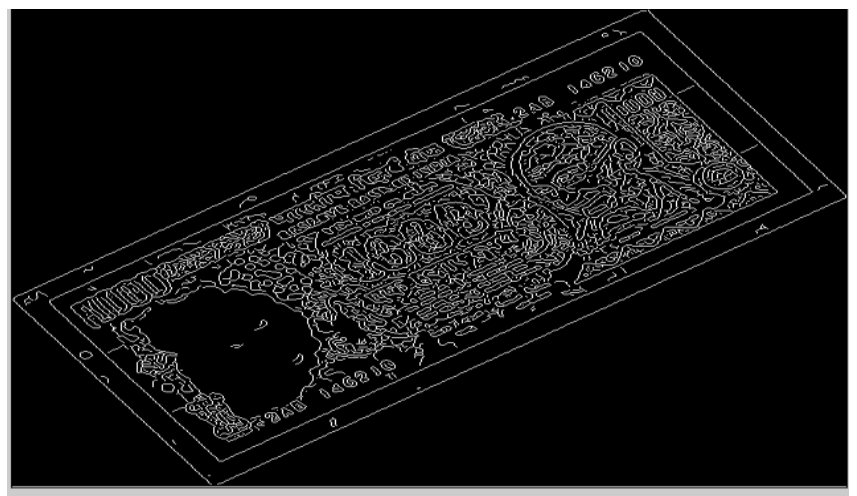

Figure 2: after canny edge

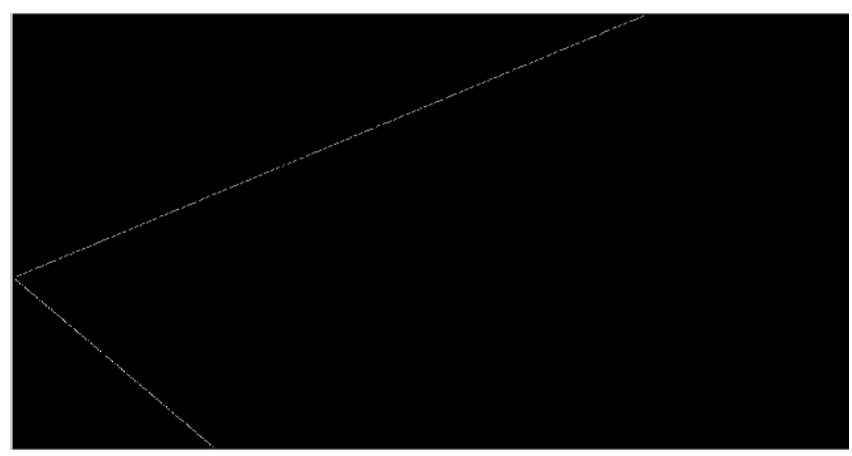

Figure 3: showing edges found by algorithm.

On setting the criterion

$$
\begin{aligned}
& \text { Select point }(\mathrm{x}, \mathrm{y}) \\
& \text { If } x>(x+1) \\
& \text { And } y>(y+1)
\end{aligned}
$$

We will get a single line as in fig 4 .

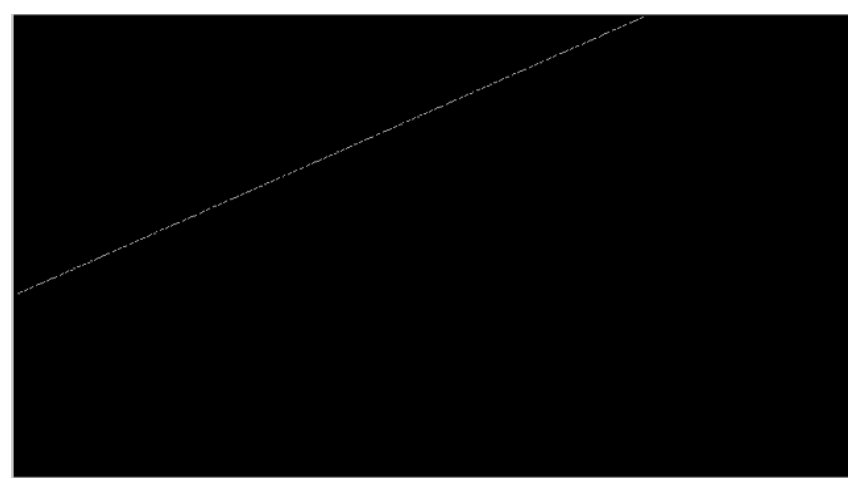

Figure 4: after putting criteria

Now, the equation of this line can be found out with the known given pts obtained using the simple mathematical formula $\theta=\tan ^{-1}\left(\frac{y 2-y 1}{x 2-x 1}\right)$ 
Due to the limitation of the canny edge detection algorithm the line obtained will be having sometimes adjacent pixels with same y coordinates and sometimes adjacent pixels different tangent as shown in fig5.

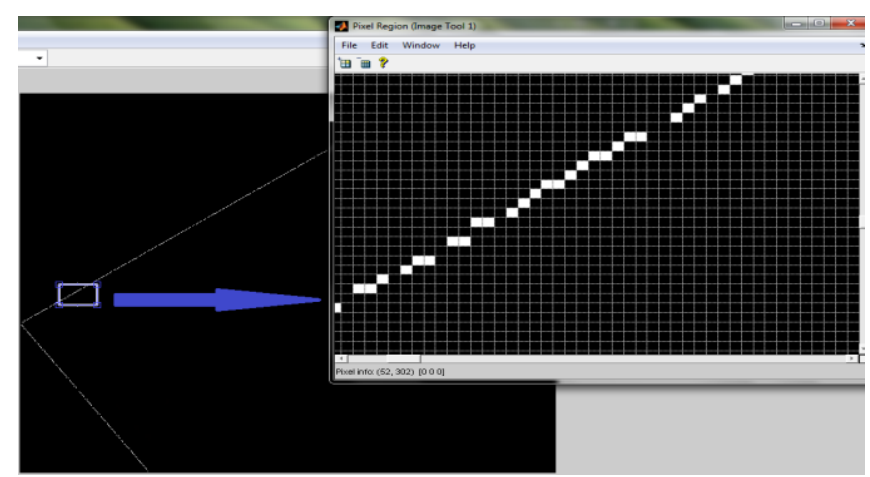

Figure 5: Edges found by canny edge detection algorithm.

For this reason the consecutive points are not taken as $\mathrm{x} 2, \mathrm{x} 1$ or $\mathrm{y} 2, \mathrm{y} 1$ instead a number of $\mathrm{dx}\left(m=\frac{d y}{d x}\right)$ and $\mathrm{dy}$ is obtained between 3 or 5 points apart and then mode $\mathrm{dx}$ (and dy corresponding to $\mathrm{dx}$ ) the tangent very frequent, is used to calculate angle.

1. b) Pre-processing for background removal: First the given note is adjusted using the angle obtained above. Then canny edge detection algorithm is applied, Again starting from the upper left corner for every row the location of the $1^{\text {st }}$ non-zero pixel is obtained. Similarly we can find upper line of the note. Thus, we get the coordinates of the edges of the note. Based on these locations we can extract out all the background pixels. The result we got are shown in fig 6 .

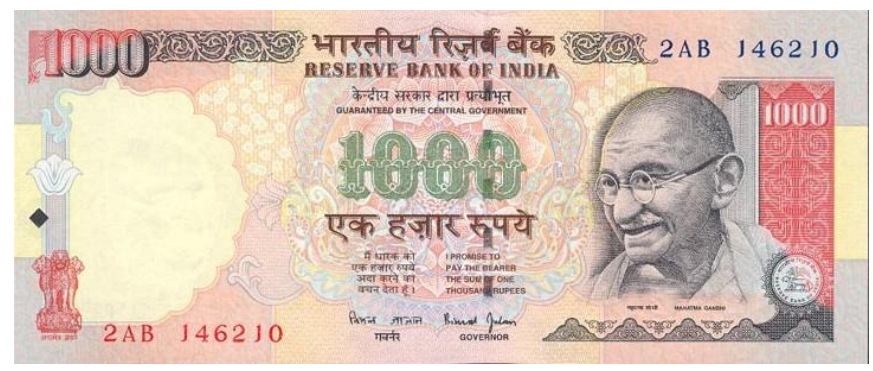

Figure 6: image without any background as compared to Figure 1.

\subsection{Pre-processing for scale invariance:}

We had rescaled the given currency note of any size to size of $512 * 512$ by interpolation techniques line nearest neighbor.

\section{Cross-Correlation of given note with sample templates:}

We had taken different templates of notes like $10,50,100,500,1000$ by cropping their corresponding pixels from corresponding images of size $512 * 512$.

Instead of calculating the conventional cross-correlation we can first approximate the template using a polynomial since
According to the Stone-Weierstrass theorem [17], any 2-D continuous function defined in closed intervals can be approximated by a polynomial with two variables. Image approximation using Legendre polynomial:

The Legendre polynomial [5] of degree $\mathrm{n}$ is defined as

$$
p^{n}(x)=\sum_{k=0}^{n / 2}(-1)^{k} \frac{(2 n-2 k) ! x^{(n-2 k)}}{2^{n} k !(n-k) !(n-2 k) !}
$$

These polynomials are orthogonal functions and are defined in the interval $-1<=\mathrm{x}<=1$. As Equation (2) shows that $P^{t 1}(x)$ has only even powers of $x$ for even $n$, and odd powers off odd $n$. It is known that the following formulae are satisfied:

$$
\begin{gathered}
p^{0}(x)=1 \\
p^{1}(x)=x \\
p^{n+1}(x)=\frac{2 n+1}{n+1} x p^{n}(x)-\frac{n}{n+1} p^{n-1}(x)
\end{gathered}
$$

The set $\mathrm{P}^{\mathrm{n}}(\mathrm{x})$ is orthogonal because it satisfies the following equations

$$
\int_{-1}^{1} p^{m}(x) p^{n}(x) d x=\left\{\begin{array}{c}
\frac{2}{2 n+1},(m=n) \\
0, \text { otherwise }
\end{array}\right.
$$

Since the set is also a complete system, any function $\mathrm{h}(\mathrm{x})$ defined in $-1<=\mathrm{x}<=1$ can be expanded by the Legendre polynomials can be defined as

$h(x)=\sum_{n=0}^{\infty} c_{n} p^{n}(x)$

$c_{n}=\frac{2 n+1}{2} \int_{-1}^{1} h(x) p^{n}(x) d x$

If we rewrite (2) as

$p^{n}(x)=\sum_{k=0}^{n} p^{n k} x^{k}$

We can calculate the coefficients $p^{n k}(0 \leq n, 0 \leq k \leq n)$ with the following recurrent formulae: 


$$
\left\{\begin{array}{l}
p^{00}=1 \\
p^{10}=0 \\
p^{11}=1 \\
p^{(n+1) k}=\frac{2 n+1}{n+1} p^{n(k-1)}-\frac{n}{n+1} p^{(n-1) k}(n \geq 1)
\end{array}\right.
$$

Where $p^{n k}=0(k<0, n<k)$ given

image $z(i, j)\left(0 \leq i \leq M_{t}-1,0 \leq j \leq N_{t}-1\right)$, first $z(i, j) \quad$ is approximated with the polynomial $h(x, y)$ of degree d, defined in

$-1 \leq x \leq 1,-1 \leq y \leq 1$, as

$$
h(x, y)=\sum c_{0 \leq m+n \leq d} p^{m}(x) p^{n}(y)
$$

Where

$c_{m n}=\frac{(2 m+1)(2 n+1)}{M_{t} N_{t}} * \sum_{i=0}^{M_{t-1}} \sum_{j=0}^{N_{t-1}} z(i, j) p^{m}\left(\frac{z_{i}}{M_{t}}-1\right) p^{n}\left(\frac{z_{j}}{N_{t}}-1\right)$

Now we can approximate the image $z(x, y)$ by the polynomial

$f(x, y)$ defined in $0 \leq x \leq 1,0 \leq y \leq 1$ as

$f(x, y)=h(2 x-1,2 y-1)$

$f(x, y)=\sum_{0 \leq m+n \leq d} c_{m n} p^{m}(2 x-1) p^{n}(2 y-1)$

$f(x, y)=\sum c_{0 \leq m n+n \leq d} \sum_{k=0}^{m} \sum_{i=0}^{n} p^{m k}(2 x-1)^{k} p^{n i}(2 y-1)^{i}$

Now we can calculate the normalized cross-correlation of $f(x, y)$ and given input image as

$$
\gamma(u, v)=\sum_{x, y} \frac{\left[f(x, y)-f^{\prime}(u, v)\right]\left[t(x-u, y-v)-t^{\prime}\right]}{\left\{\sum_{x, y}\left[f(x, y)-f^{\prime}(u, v)\right]^{2} \sum_{x, y}\left[t(x-u, y-v)-t^{\prime}\right]^{2}\right\}^{0.5}}
$$

Where $f$ is the image

$t$ is mean of the image.

$f^{\prime}(u, v)$ Is the mean of the $f(x, y)$ in the region under the template.

\section{CLASSIFICATION:}

We can classify the currency as one having maximum normalized cross-correlation score of its template with the given input image.

\section{RESULTS AND CONCLUSION:}

Our approach aimed at improving the existing system's which are solving the problem of currency classification, once the currency is being pre-processed with the help of methods which are being discussed in the above sections, we get image in simplest form. Once we are having the image then for classification we can adopt any approach like neural network or template matching. The system efficiency increases manifold, if we are successfully able to make our system rotational invariant and able to remove the background. In this part we are applying template matching for the classification of the currency values, the results after the template matching are shown in the fig 6 .

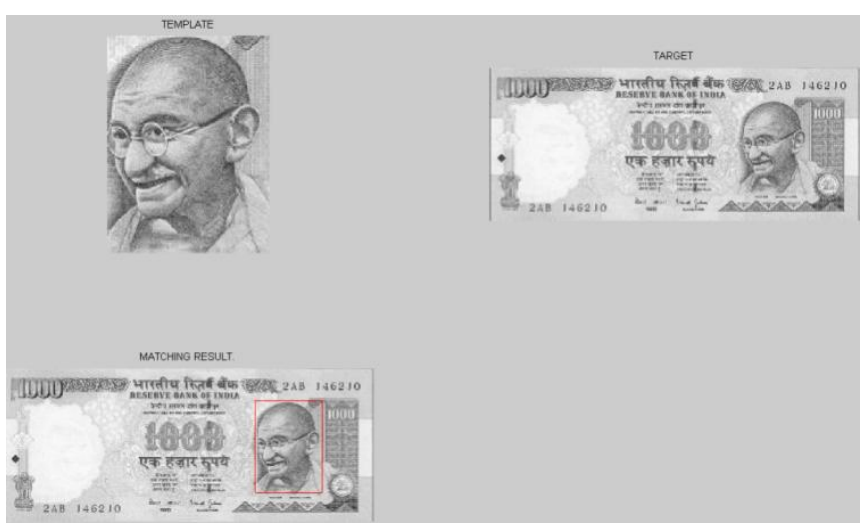

Figure 6. Template matched is shown in red box.

Below are the results of rotation invariant algorithm

Table 2:

\begin{tabular}{|l|l|}
\hline $\begin{array}{l}\text { Angle introduced for } \\
\text { Experimenting. }\end{array}$ & Angle found by the Algorithm. \\
\hline 16 & 16.5 \\
30 & 29.7 \\
44 & 44 \\
60 & 59.6 \\
74 & 73.1 \\
88 & 86.9 \\
\hline
\end{tabular}


For the current working we have only limited our system to template matching, in the future working neural network approaches can also be applied for efficiently classifying the images. By considering rotation and background image and providing useful algorithm for their correction, we can say that our system will perform more efficiently in real world scenario.

\section{REFERENCES:}

[1] F. Takeda and S. Omatu. "Bank note recognition system using neural network with random masks", Proc. World Cong. Neural Net., Portland, OR, 1: 241-244,1993.

[2]F. Takeda and S. Omatu. "High speed paper currency recognition by neural networks", EEE Trans. Neural Networks, 6(1):73-77,1995.

[3]F. Takeda, S. Omatu and S. Onami. "Mask optimization by genetic algorithm for a neuro pattern recognition machine with masks", Trans. Inst. of Syst. Contr. and Inform. Engineers, 8(5): 196203, 1995.

[4]S. Pattaramalai, P. Pimsen and K. Chamnongthai. "A Thainote recognition by using two frequency bands and neural network", Proc. IASTED lnt. Conf. Modelling, Simulation and Optimization, 16-19, 1997.

[5] G. B. Arfken and H. J. Weber, Mathematical Methods for Physicists,6th ed. New York: Academic, 2005.

[6]M. Prakash and M. Narasimha Murty. "Growing subspace pattern recognition methods and their neural-network models", IEEE Trans. Neural Network, 8(1): 161-168, 1997.

[7]C.M. Bishop. "Neural Netowork for Pattern Recognition",

Clarendon Press.Oxford. 1995..
[8] A. Frosini, M. Gori and P. Priami. "A neural network-based model for paper currency recognition and verification", IEEE. Trans. Neural Networks,7(6): 1482-1490,1996.

[9] M. Bianchini, P. Frasconi and M. Gori. "Learning in multilayered networks used as autoassociators", IEEE Trans. Neural Networks, 6(2): 512-515, 1995.

[10] Bazil Shaik and Sandhya Srinivasan, ( 'The Paper and the Promise: A Brief History of Currency and Bank Notes in India', Reserve Bank of India. 2001).

[11] Balachandran, G.), 'The Reserve Bank of India, 1951-67 ', Oxford University Press. (1998).

[12] A. Rosenfeld and G. J. VanderBrug, "Coarse-fine template matching," IEEE Trans. Syst., Man. Cybern., vol. SMC-7, no. 2, pp. 104-107, Feb. 1977.

[13] G. J. VanderBrug and A. Rosenfeld, "Two-stage template matching," IEEE Trans. Comput., vol. C-26, no. 4, pp. 384-393, Apr. 1977.

[14] W. H. Press, B. P. Flannery, S. A. Teukolsky, and W. T. Vetterling, Numerical Recipes in C: The Art of Scientific Computing, 2nd ed. Cambridge, U.K.: Cambridge Univ. Press, 1992.

[15] H. Schweitzer, J. W. Bell, and F. Wu, "Very fast template matching," in Proc. 7th Eur. Conf. Computer Vision IV, 2002, pp. 358-372.

[16]"Shinichiro Omachi, Member, IEEE, and Masako Omachi "Fast Template Matching With Polynomials".IEEE TRANSACTIONS ON IMAGE PROCESSING, VOL. 16, NO. 8, AUGUST 2007.

[17] R. M. Dudley, Real Analysis and Probability. Cambridge, U.K.: Cambridge Univ. Press, 2002. 